Original article

\title{
Can Generalized Poisson model replace any other count data models? An evaluation
}

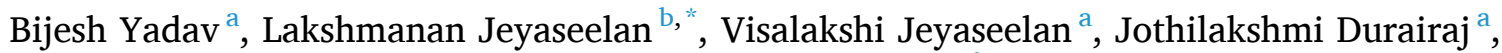 \\ Sebastian George ${ }^{c}$, K.G. Selvaraj ${ }^{\text {a }}$, Shrikant I. Bangdiwala ${ }^{d}$

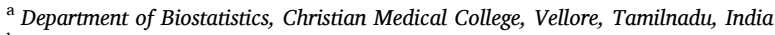 \\ ${ }^{\mathrm{b}}$ Mohammed Bin Rashid University of Medicine and Health Sciences, Dubai Heathcare City, Dubai, United Arab Emirates \\ ${ }^{\mathrm{c}}$ Department of Statistical Sciences, Kannur University, Kannur, Kerala, India \\ ${ }^{\mathrm{d}}$ Population Health Research Institute and Department of Health Research Methods, Evidence and Impact, McMaster University, Hamilton, ON, Canada
}

\section{A R T I C L E I N F O}

\section{Keywords:}

Over-dispersion

Mixture distribution

Generalized Poisson

Count data

Simulation

\begin{abstract}
A B S T R A C T
Background: Count data represents the number of occurrences of an event within a fixed period of time. In count data modelling, overdispersion is inevitable. Sometimes, this overdispersion may not be just due to the excess zeros but may be due to the presence of two or more mixtures. Hence the main objective is to examine for the presence of mixtures if any, with excess zeros and compare Generalized Poisson model, Mixture models with other count data models using real time and simulated data.

Methods: Three real time over-dispersed datasets were used for the comparison of the models. The real time data models were compared using information criteria like AIC and BIC and regression coefficients. Data was also simulated using mixture Poisson with excess zeros. The simulation was repeated for different sample sizes were used to identify the better model.

Results: Generalized Poisson showed consistently lower bias and MSE when compared to the other models for varying sample of sizes. AIC and BIC values were almost similar for Generalized Poisson, ZIP and Mixture Poisson model. Similar findings were also obtained from real time data.

Conclusion: Generalized Poisson models provides a better fit for overdispersed data due to excess zeros, consistently in real time and simulated with varying sample sizes. Negative Binomial models can be redistricted or reevaluated against Generalized Poisson model.
\end{abstract}

\section{Introduction}

Count data represents the number of occurrences of an event within a fixed period of time (Examples: Number of an Antenatal care (ANC) visits, Number of postoperative complications, Number of alcohol drink past one months, Number of doctor visits etc.). Modelling count data is a common task in epidemiological, social and economic fields. Poisson and Negative Binomial models are used frequently to analyze count data. In many situations over dispersion is more frequent issue to be dealt with count data. Poisson distribution constrained by its equidispersion assumption, making it less than ideal for modelling real data that often exhibit over-dispersion or under-dispersion. Analysis of count data that are over dispersed relative to the Poisson distribution has been studied extensively, as the standard Poisson model may result in misleading inferences. In count data modelling, overdispersion is usually meant to occur when there are excess zeros, Sometimes, this overdispersion may not be just due to the excess zeros but may be due to the presence of two or more mixtures distribution. ${ }^{1}$ When there is overdispersion, Zero Inflated Poisson (ZIP) models are recommended.

Finite mixture models are appropriate when the extra variability comes from the unobserved heterogeneity of the population, which composes two or more subgroups mixed in various proportions. ${ }^{2-5}$ Count data mixture models often deal with extra dispersion problems especially in medical research where data is often count.

In particular, Poisson mixture regression can be fitted to such extra dispersed count data in the presence of covariate information. ${ }^{6} \mathrm{~A}$ mixture Poisson regression model assumes that the interpretations are drawn from a finite mixture Poisson where these distributions differ in

\footnotetext{
* Corresponding author. Mohammed Bin Rashid University of Medicine and Health Sciences, Dubai, PO Box 505055, United Arab Emirates.

E-mail addresses: yadavbijesh@gmail.com (B. Yadav), ljey@hotmail.com (L. Jeyaseelan), visali_pv@hotmail.com (V. Jeyaseelan), jothidurairaj72@gmail.com (J. Durairaj), sthottom@gmail.com (S. George), selvarajcmc@gmail.com (K.G. Selvaraj), bangdiws@mcmaster.ca (S.I. Bangdiwala).
} 
intercept, and coefficient of the explanatory variable in the regression component. ${ }^{6}$ When there is a mixture of two or more Poisson or Negative Binomial distribution, it implies that count distribution is more skewed. The Generalized Poisson Distribution (GPD) includes the Poisson distribution as a special case, and over the range where the second parameter is positive, it is overdispersed relative to Poisson with a variance to mean ratio exceeding one. ${ }^{7}$ For the case of both overdispersed or underdispersed count data, Generalized Poisson and Conway-Maxwell-Poisson (COM) regression models have been suggested and fitted by several researchers. ${ }^{8-15}$ GPD is two parameters $(\theta, \delta)$ distributions that generalize the usual Poisson distribution in a flexible manner. By changing $\delta$, it is possible to induce an increase or decrease in the occurrence rate being modelled. GPDs arise in several discrete models where the probabilities depend either on the previous number of occurrences or on the average number of events in any given range. Thus, the generalized Poisson distribution becomes a useful model for counting processes where the occurrence of events is not homogeneous. ${ }^{16}$ However, there has been no formal review of GPD in relation to mixture regression and zero-inflated models done so far. Hence the main objective is to examine for the presence of mixtures if any, and compare Mixture Poisson, Mixture Negative Binomial, Zero-inflated Poisson and Generalized Poisson models in terms of bias, mean square error, AIC and BIC using real time and simulated data.

\section{Methods}

\subsection{Poisson model}

The most commonly used regression model for count data is the Poisson regression model, where covariates are included in the model via an invertible link function describing the relationship of the linear predictor $x_{i} \beta=\eta_{i}$ to the expected value of the responses $\theta_{\mathrm{i}}$ :

$f\left(y_{i}, \theta_{i}\right)=\frac{\theta_{i}^{y_{i}} e^{-\theta_{i}}}{y_{i} !}, y_{i}=0,1,2, \ldots, \theta_{i}>0$

The expected outcome in terms of the inverse of the log link function is given by $\theta_{i}=\exp \left(x_{i} \beta\right)$, where $x_{i}$ is a covariate vector and $\beta$ is a vector of regression parameters to be estimated. ${ }^{17}$

\subsection{Generalized Poisson model}

For equidispersed or for overdispersed or underdispersed count data $\mathrm{Y}$, we may consider a regression model based on the GP distribution. This model assumes the response variable $Y_{i}$ has probability mass function (PMF)

$f\left(y_{i}, \theta_{i} \delta\right)=\frac{\theta_{i}\left(\theta_{i}+\delta y_{i}\right)^{y_{i}-1} \mathrm{e}^{-\theta_{i}-\delta y_{i}}}{y_{i} !}, y_{i}=0,1,2, \ldots$

Where $\theta_{\mathrm{i}}>0$ and $\max \left(-1,-\theta_{\mathrm{i}} / 4\right)<\delta<1$. The mean and variance of the GP random variable $Y_{i}$ are given by

Mean $\left(\mu_{\mathrm{i}}\right)=E\left(Y_{i}\right)=\frac{\theta_{i}}{1-\delta}$
$\operatorname{Variance}\left(Y_{i}\right)=\frac{\theta_{1}}{(1-\delta)^{3}}=\frac{1}{(1-\delta)^{2}} E\left(Y_{i}\right)=\varphi E\left(Y_{i}\right)$

The term $\varphi=1 /(1-\delta)^{2}$ plays the role of a dispersion factor. Clearly, when $\delta=0$, there is equidispersion, and the GP distribution reduces to the usual Poisson distribution with parameter $\theta_{\mathrm{i}}$. Further, when $\delta>0$, we have overdispersion in the model; when $\delta<0$, we have underdispersion. ${ }^{17}$

\subsection{Mixture models}

In analysis of data, often there are heterogeneous sub populations found within the main population. In such cases use of a single distribution will not accurately model the data.
It requires modeling the sub populations in order to appropriately model the entire population.

The idea of a 'mixture of models' is used to model the populations with heterogeneous sub populations. A mixture model is a probabilistic model for representing the presence of subpopulations within an overall population, without requiring that an observed data set should identify the sub-population to which an individual observation belongs.

The probability function of the data with $\mathrm{K}$ sub populations is given as

$f(x, \theta)=\sum_{k=1}^{k} p_{k} f_{k}\left(x, \theta_{k}\right)$

Where $\theta$ denotes the set of parameters of the mixture model to be estimated and $\mathrm{p}_{\mathrm{k}}$ the weight of each probability density function that satisfies $\sum_{k=1}^{k} p_{k}=1$ and $0 \leq \mathrm{pk} \leq 1$.

Sometimes the number of sub populations in a population is not a known number. In such cases where the number of sub populations is unknown, the problem leads to an interesting instance of 'estimation of mixture components' which adds extra complexity to the problem. When the number of mixture components is known and finite the problem leads to a "Finite Mixture Model". The weight of each probability density function $\mathrm{p}_{\mathrm{k}}$ is also known as the mixture probability. Mixture probability denotes the probability of the observations belonging to each sub population. This being a value between 0 and 1 the $\mathrm{p}_{\mathrm{k}}$ could be a constant or an expression of a variable of interest.

\subsection{Zero-inflated models}

Zero-inflated model is two-component or mixture model (mixture of two distributions).

For rare events or event counts with low means, both the standard Poisson and Negative Binomial (NB) models can actually predict a high proportion of zeros, with NB being able to predict more zeros. However, when data have "excessive" zeros-far more than what can be accounted for by either the Poisson or the NB distribution, neither the standard Poisson nor the NB models is adequate. In these cases, adjusting for extra-zeros becomes necessary. The zero-inflated Poisson (ZIP) model is one of these adjustments. ${ }^{18}$ The ZIP model assumes that data are generated from two different processes: one process generates only the zero count (structured zeros, e.g., non-smokers) from the always-zero group (non-risk group), and another process generates both the zero (random zeros, e.g., smokers who did not smoke on a particular day) and non-zero counts from the not-always-zero group (at-risk group). Accordingly, ZIP combines the two modeling processes: the logistic regression portion predicts the presence or absence of the outcome due to structured zeros, and the Poisson regression portion predicts the extent of the count outcome, which may include zero counts (random zeros) too.

The probability density function (PDF) of the zero-inflated Poisson model defined as

$\operatorname{pr}\left(Y_{i}=y_{i} \mid x_{i}, z_{i}\right)=\left\{\begin{array}{l}p_{i}+\left(1-p_{i}\right) e^{-\lambda_{i}}, y_{i}=0 \\ \left(1-p_{i}\right) \frac{e^{-\lambda_{i}} \lambda_{i}^{y_{i}}}{y !}, y_{i}>0\end{array}\right.$

Where $\lambda_{\mathrm{i}}$ is still the Poisson mean, and $\mathrm{X}_{\mathrm{i}}$ and $\mathrm{Z}_{\mathrm{i}}$ are covariate vectors for the zero-inflation and Poisson portions of the model, respectively. The covariates for each part (logistic and Poisson) of the model, $\mathrm{X}_{\mathrm{i}}$ and $\mathrm{Z}_{\mathrm{i}}$, can be different or the same. $P_{i}$ is the probability of extra zeros.

When the probability of extra zero counts becomes nil $\left(P_{i}=0\right)$, the ZIP model reduces to a regular Poisson model. ${ }^{19}$ 


\subsection{Model selection}

\subsubsection{Akaike information criteria (AIC) and Bayesian Schwartz information criteria (BIC)}

When several maximum likelihood models are available, one can compare the performance of alternative models based on several likelihood measures which have been proposed in the statistical literature. Two of most regularly used measures are the AIC and BIC. The AIC is defined as

$\mathrm{AIC}=-2 \mathrm{~L}+2 \mathrm{k}$

Where, $\mathrm{L}$ denotes the log-likelihood function and $\mathrm{k}$ is the number of estimated parameters included in the model (number of variables plus the intercept). The smaller the AIC, the better the model.

The BIC is defined as

$\mathrm{BIC}=-2 \mathrm{~L}+\mathrm{k} \log (\mathrm{n})$

Where, $\mathrm{L}$ denotes the log-likelihood, $\mathrm{k}$ the number of parameters, including intercept and $\mathrm{n}$ the number of rating classes or number of model observations. The smaller the BIC value, the better is the model.

\subsection{Data simulation}

An independent variable $(\mathrm{X})$ was generated from uniform distribution $\mathrm{U}(0,1)$ of $\mathrm{n}$ observations. Using that independent variable, the dependent variable was generated as a linear combination with fixed intercept $(a=0.01)$ and slope $(b=0.03)$ (which is assumed as the population mean). A low value of beta was chosen so as to generate more zeros. The antilog of the predicted value from the regression equation was the mean. This mean was used to generate two Poisson mixtures with mixing proportion of 0.6 and 0.4 respectively. Once the data was generated, the mixture Poisson regression model was applied to the simulated data using Flexmix package using R. ZIP model, Generalized Poisson models were also applied. The comparison of the models was done by calculating MSE, Bias and information criteria such as AIC, BIC. Bias was defined as the difference between populations mean and expected which was obtained from the simulated data. MSE was the square of deviation of all simulated samples from the population mean. When there is less bias, MSE, AIC and BIC values are also small. So it was expected that better model was the model with lower AIC, BIC, Bias and MSE. This whole process was repeated 1000 times and the mean Bias, MSE are reported for varying sample sizes.

\subsection{Real time data}

The dataset "Number of doctor visits" taken from the U.S. Medical Expenditure Panel Survey for 2003 which talks about the number of children a couple has, the number of doctor's visits per year a person makes, and the number of trips per month that a person takes? The aim of the study is to find the factors influencing the number of doctor visits. ${ }^{20}$ The dataset "Marital Status Inventory (MSI)" taken from online. The MSI assesses a spectrum of behaviours related to relationship separation (e.g., "Thoughts of divorce occur to me fairly frequently, about once a week or more"). ${ }^{21}$ The data "Number of injections in last 12 months" obtained from the cross-sectional survey conducted by National Family Health Survey (NFHS-3) in 2005-2006. The National Family Health Surveys (NFHS) are nationwide surveys conducted with a representative sample of households throughout the country by the Ministry of Health and Family Welfare (MOHFW), Government of India. The report shows that women are somewhat more likely than men to have received at least one injection (39\% and 36\%, respectively) given by health personnel in the 12 months preceding the survey. From this data, we have randomly selected (1274) of the women's data. ${ }^{22}$

\section{Results}

Data was simulated with different sample sizes 50, 100, 400, 500, 600 and 1000 . The results of simulation showing the Bias, MSE, AIC and BIC are presented in Table 1.

Sample Size 50: The bias obtained from the Mixture Poisson, Mixture Negative Binomial, ZIP and Generalized Poisson was found to be 6.53, 6.53, 7.84 and 6.34 respectively. The MSE for Mixture Poisson, Mixture Negative Binomial, Zero-inflated Poisson and Generalized Poisson was found to be 172.37, 172.64, 192.17 and 170.52 respectively. The AIC and BIC for Mixture Poisson, Mixture Negative Binomial, Zero-inflated Poisson and Generalized Poisson was found to be 158 and 167, 168 and 178, 156 and 164, and 156 and 162 respectively.

Sample Size 100: The bias and MSE for a sample size of 100 was found to be 6.21 and 317.21 for mixture Poisson model, 5.88 and 312.35 for mixture Negative Binomial model, 6.97 and 328.62 for ZIP model, and 5.88 and 312.35 for Generalized Poisson model. Similarly, the AIC and BIC was found to be 280 and 293 for mixture Poisson model, 293 and 306 for mixture Negative Binomial model, 279 and 289 for ZIP model, and 280 and 288 for Generalized Poisson model.

Sample Size 400: The bias and MSE for a sample size of 400 was found to be 4.53 and 150.10 for mixture Poisson model, 4.53 and 150.10 for mixture Negative Binomial model, 8.93 and 210 for ZIP model, and 4.46 and 150.08 for Generalized Poisson model. Similarly, the AIC and BIC was found to be 1053 and 1073 for mixture Poisson model, 1109 and 1129 for mixture Negative Binomial model, 1051 and 1067 for ZIP model, and 1049 and 1061 for Generalized Poisson model.

Sample Size 500: The bias obtained from the Mixture Poisson, Mixture Negative Binomial, ZIP and Generalized Poisson was found to be 4.35, 4.35, 18.12 and 4.38 respectively. The MSE for Mixture Poisson, Mixture Negative Binomial, Zero-inflated Poisson and Generalized Poisson was found to be 130.81, 130.81, 490.92 and 131.2 respectively. The AIC and BIC for Mixture Poisson, Mixture Negative Binomial, Zeroinflated Poisson and Generalized Poisson was found to be 1322 and 1343, 1426 and 11447, 1320 and 1337, and 1316 and 1329 respectively.

Sample Size 600: The bias obtained from the Mixture Poisson,

Table 1

Comparison of Bias, MSE, AIC and BIC based on simulated data.

\begin{tabular}{|c|c|c|c|c|}
\hline Models & Bias & MSE & AIC & BIC \\
\hline \multicolumn{5}{|l|}{$\mathbf{N}=\mathbf{5 0}$} \\
\hline Mixture Poisson & 6.53 & 172.37 & 158 & 167 \\
\hline Mixture Negative Binomial & 6.53 & 172.64 & 168 & 178 \\
\hline Zero-inflated Poisson & 7.84 & 192.17 & 156 & 164 \\
\hline Generalized Poisson & 6.34 & 170.52 & 156 & 162 \\
\hline \multicolumn{5}{|l|}{$N=100$} \\
\hline Mixture Poisson & 6.21 & 317.21 & 280 & 293 \\
\hline Mixture Negative Binomial & 5.88 & 312.35 & 293 & 306 \\
\hline Zero-inflated Poisson & 6.97 & 328.62 & 279 & 289 \\
\hline Generalized Poisson & 5.88 & 312.35 & 280 & 288 \\
\hline \multicolumn{5}{|l|}{$N=400$} \\
\hline Mixture Poisson & 4.53 & 150.10 & 1053 & 1073 \\
\hline Mixture Negative Binomial & 4.53 & 150.10 & 1109 & 1129 \\
\hline Zero-inflated Poisson & 8.93 & 210.32 & 1051 & 1067 \\
\hline Generalized Poisson & 4.46 & 150.08 & 1049 & 1061 \\
\hline \multicolumn{5}{|l|}{$\mathbf{N}=\mathbf{5 0 0}$} \\
\hline Mixture Poisson & 4.35 & 130.81 & 1322 & 1343 \\
\hline Mixture Negative Binomial & 4.35 & 130.81 & 1426 & 1447 \\
\hline Zero-inflated Poisson & 18.12 & 440.92 & 1320 & 1337 \\
\hline Generalized Poisson & 4.38 & 131.20 & 1316 & 1329 \\
\hline \multicolumn{5}{|l|}{$N=600$} \\
\hline Mixture Poisson & 7.21 & 125.21 & 1652 & 1674 \\
\hline Mixture Negative Binomial & 7.21 & 125.26 & 1744 & 1766 \\
\hline Zero-inflated Poisson & 9.77 & 130.95 & 1650 & 1668 \\
\hline Generalized Poisson & 7.00 & 125.46 & 1644 & 1664 \\
\hline \multicolumn{5}{|l|}{$\mathbf{N}=1000$} \\
\hline Mixture Poisson & 4.38 & 142.46 & 2649 & 2674 \\
\hline Mixture Negative Binomial & 4.38 & 142.46 & 2803 & 2827 \\
\hline Zero-inflated Poisson & 7.55 & 180.17 & 2645 & 2665 \\
\hline Generalized Poisson & 4.34 & 142.11 & 2645 & 2660 \\
\hline
\end{tabular}


Mixture Negative Binomial, ZIP and Generalized Poisson was found to be 7.21, 7.21, 9.77 and 7.00 respectively. The MSE for Mixture Poisson, Mixture Negative Binomial, Zero-inflated Poisson and Generalized Poisson was found to be $125.21,125.26,130.95$ and 125.46 respectively. The AIC and BIC for Mixture Poisson, Mixture Negative Binomial, Zero-inflated Poisson and Generalized Poisson was found to be 1652 and 1674, 1744 and 1766, 1650 and 1686, and 1644 and 1664 respectively.

Sample Size 1000: The bias and MSE for a sample size of 1000 was found to be 4.38 and 142.46 for mixture Poisson model, 4.38 and 142.46 for mixture Negative Binomial model, 7.55 and 180.17 for ZIP model, and 4.34 and 142.11 for Generalized Poisson model. Similarly, the AIC and BIC was found to be 2649 and 2674 for mixture Poisson model, 2803 and 2827 for mixture Negative Binomial model, 2645 and 2665 for ZIP model, and 2645 and 2660 for Generalized Poisson model.

Summary for simulated data: The bias when compared across the models, Generalized Poisson showed low values compared to all other models except when the sample size was 500. The MSE values consistently smaller in Generalized Poisson compared with other models across the sample sizes. The AIC and BIC values were almost similar for Generalized Poisson, Zero Inflated model and Mixture Poisson model. Generalized Poisson consistently provides lower estimates for all parameters in different sample sizes.

The result from the real time data sets is presented in Table 2. The distribution of the data is presented in Fig. 1. The proportion of zero counts from "visits to doctor ( $=3677)$ ), "Marital Status Inventory (N = 263)" and "Number of injections in last 12 months $(\mathrm{N}=1274)$ " data was found to be $10.9 \%, 24 \%$ and $66 \%$ respectively. The above three datasets were over-dispersed due to excess zeros based on Poisson model. The mean counts with variance was found to be 6.82(54.69), $3.19(7.21)$ and 1.58 (18.43) of the datasets "visits to doctor", "Marital Status Inventory" and "Number of injections in last 12 months" respectively.

Number of doctor visits: The regression coefficient for age from "visits to doctor" data when mixture Poisson model was applied was 0.01 "component 1 " and 0.0008 "component 2" while mixture Negative Binomial showed 0.003 (component 1) and 0.014 (component 2). ZIP

Table 2

Comparison of Regression coefficient, AIC and BIC based on real time data.

\begin{tabular}{|c|c|c|c|c|c|c|}
\hline \multirow[t]{2}{*}{ Models } & \multicolumn{2}{|c|}{$\begin{array}{l}\text { Regression } \\
\text { Coefficients }\end{array}$} & \multicolumn{2}{|c|}{ Standard errors } & \multirow[t]{2}{*}{ AIC } & \multirow[t]{2}{*}{ BIC } \\
\hline & Comp.1 & Comp.2 & Comp.1 & Comp.2 & & \\
\hline \multicolumn{7}{|c|}{ Number of doctor visits $(\mathrm{N}=3677)$} \\
\hline Mixture Poisson & 0.01 & 0.0008 & 0.002 & 0.002 & 24884 & 24884 \\
\hline $\begin{array}{l}\text { Mixture } \\
\text { Negative } \\
\text { Binomial }\end{array}$ & 0.003 & 0.014 & 0.002 & 0.002 & 21990 & 22021 \\
\hline $\begin{array}{l}\text { Zero-inflated } \\
\text { Poisson }\end{array}$ & 0.004 & -0.033 & 0.0001 & 0.009 & 31225 & 31250 \\
\hline $\begin{array}{c}\text { Generalized } \\
\text { Poisson }\end{array}$ & 0.01 & & 0.002 & & 21949 & 21968 \\
\hline \multicolumn{7}{|c|}{ Marital Status Inventory $(N=263)$} \\
\hline Mixture Poisson & 0.45 & -0.16 & 0.60 & 0.08 & 1160 & 1178 \\
\hline $\begin{array}{l}\text { Mixture } \\
\text { Negative } \\
\text { Binomial }\end{array}$ & -0.175 & -0.173 & 0.10 & 0.08 & 1219 & 1236 \\
\hline $\begin{array}{l}\text { Zero-inflated } \\
\text { Poisson }\end{array}$ & -0.19 & -0.09 & 0.07 & 0.30 & 1172 & 1186 \\
\hline $\begin{array}{c}\text { Generalized } \\
\text { Poisson }\end{array}$ & -0.148 & & 0.11 & & 1213 & 1224 \\
\hline \multicolumn{7}{|c|}{ Number of injections in last 12 months $(N=1274)$} \\
\hline Mixture Poisson & -7.33 & 2.62 & 5.22 & 0.16 & 3515 & 3538 \\
\hline $\begin{array}{l}\text { Mixture } \\
\text { Negative } \\
\text { Binomial }\end{array}$ & -0.24 & 9.17 & 0.0002 & 8.94 & 2681 & 2704 \\
\hline $\begin{array}{l}\text { Zero-inflated } \\
\text { Poisson }\end{array}$ & -0.05 & 0.07 & 0.191 & 0.195 & 3641 & 3660 \\
\hline $\begin{array}{c}\text { Generalized } \\
\text { Poisson }\end{array}$ & -0.17 & & 0.156 & & 2677 & 2692 \\
\hline
\end{tabular}

showed 0.004 for count part and -0.003 for the logit part. Generalized Poisson regression result was 0.01 . AIC and BIC values were 24884 and 24884 for mixture Poisson model. AIC and BIC values were 21990 and 22021 for mixture Negative Binomial model. AIC and BIC values were 31225 and 31250 for ZIP. AIC and BIC values were 21949 and 21968 for generalized Poisson.

Marital Status Inventory (MSI): The regression coefficient for gender when mixture Poisson model was applied was 0.45 "component 1 " and - 0.16 "component 2" while mixture Negative Binomial showed 0.175 (component 1 ) and -0.173 (component 2). ZIP showed - 0.19 for count part and -0.09 for the logit part. Generalized Poisson regression result was - 0.148. The AIC and BIC values for MSI data were 1160 and 1178 (mixture Poisson model), 1219 and 1236 (mixture Negative Binomial), 1172 and 1186 (ZIP), 1213 and 1224 (Generalized Poisson).

Number of injections in last 12 months: The regression coefficient for tobacco use when mixture Poisson model was applied was -7.33 "component 1" and 2.62 "component 2" while mixture Negative Binomial showed - 0.24 (component 1) and 9.17 (component 2).

ZIP showed - 0.05 for count part and 0.07 for the logit part. Generalized Poisson regression result was - 0.17. The AIC values were 3515, 2681, 3641 and 2677 for mixture Poisson, mixture Negative Binomial, ZIP and Generalized Poisson respectively. The BIC values for mixture Poisson, mixture Negative Binomial, ZIP and Generalized Poisson were found to be 3538, 2704, 3660 and 2692 respectively.

Summary for real time data: The AIC and BIC were smaller in generalized Poisson model compared with other models in the real time data except MSI. The Standard errors consistently lower in generalized Poisson model.

\section{Discussion}

There are studies that compared regression coefficients, bias, MSE between GPD and NB models. ${ }^{23-25}$ However, there is a dearth of studies that compared Generalized Poisson with ZIP and Mixture Poisson models. Hence it is a genuine attempt to compare these models in terms of information criteria, Bias and MSE. A study compared regression coefficients and information criteria using Poisson, Negative Binomial and Generalized Poisson models on Antenatal care visits in Nigeria. ${ }^{23}$ The study showed that Generalized Poisson model was a better model in terms of AIC and BIC. This finding was similar to the present study but the comparison was not only based on AIC and BIC but also in terms of Bias and MSE. Another study compared only Negative Binomial with Generalized Poisson model for over-dispersion count data. The study concluded that Generalized Poisson model had significant findings based on real time fertility data which was similar to the present study finding. ${ }^{24}$ However, the present study used real time and simulated data to examine the differences across the four models. The study concluded that Generalized Poisson and Negative Binomial models had higher standard errors. The present study not only compared standard errors for the real time datasets but also generated mixture Poisson data for the comparison of four models in terms of information criteria, bias and MSE.

The simulated data was generated in such a way that the regression coefficients were less correlated with the outcome so as to produce more zeros. This was done deliberately so as to apply ZIP model to the simulated data.

The limitation of the study was that the data was simulated using two mixtures and therefore the results showed that mixture Poisson had lower bias and MSE. However, bias from generalized Poisson was lower than ZIP model and thereby our conclusion about generalized Poisson model is valid. This study compared Generalized Poisson with mixture Negative Binomial rather than Zero-inflated Negative Binomial provide similar result. Generalized Poisson model which had lower AIC and BIC values with lower MSE and bias even in the presence of over-dispersion as compared to ZIP and Mixture Poisson models. Generalized Poisson model had similar bias and MSE to mixture Poisson and mixture 


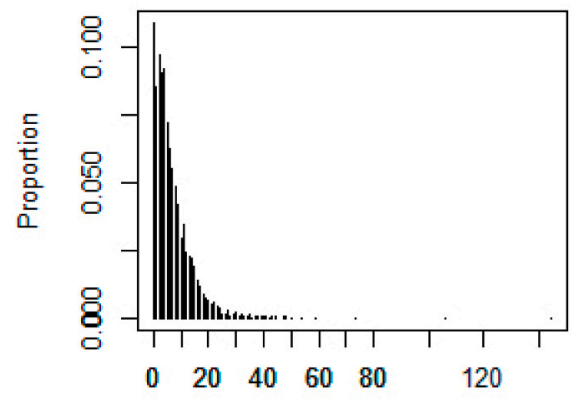

Number of Visits to Doctor

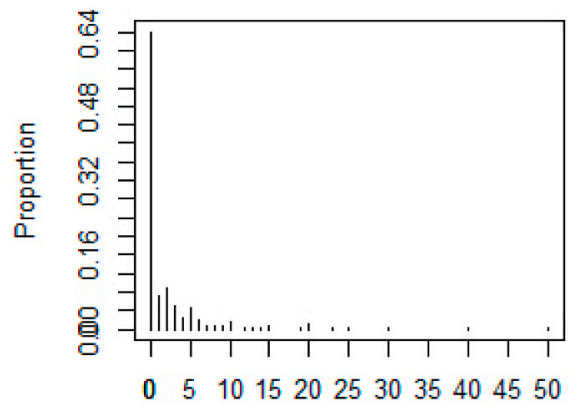

Number of injections in last 12 months

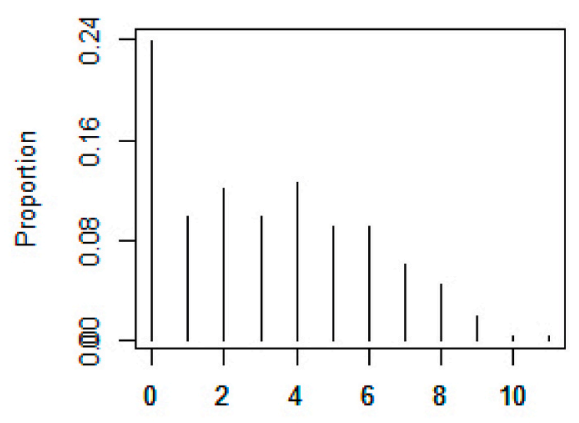

Number of Marital Status Inventory

Fig. 1. Distribution of counts from Visits to doctor, marital status inventory and Number of injections in last 12 months datasets.

Negative Binomial models when the size of 500 observations was considered. However, difference of at least 10 units in the BIC was considered as an important difference when comparing models. ${ }^{26}$ For the real time data, only age was considered for analysis as the intention was to compare the model fit in the presence of excess zeroes and not to estimate model coefficients. .

\section{Conclusion}

From this paper it is suggested that Generalized Poisson models would provide reasonably better fit for over-dispersed data due to excess zeros as compared to Zero-inflated Poisson and Mixture Negative Binomial. Negative Binomial models can be restricted or re-evaluated against generalized Poisson model. It had lower estimates in the presence of over-dispersion as compared to other models and hence considered to be a better model in general. This finding has not been reported elsewhere.

\section{Declaration of competing interest}

The author(s) declared no potential conflicts of interest with respect to the research, authorship, and/or publication of this article.

\section{Funding}

Not applicable.

\section{Contributors}

This study was conceived of and planned by all authors. BY undertook the data analysis, with statistical advice and contributions from all authors on the results and interpretation. $\mathrm{VJ}$ guided for doing simulation and writing the manuscript with contribution and editing from all authors. L.J involved in phrasing the research questions. He also involved in the verification of analytical results and drafting methods and discussion section. JD helped in simulation. SG provided his expertise regarding generalized Poisson. SB and KGS drafted the discussion and methods section along with LJ.

\section{References}

1 Harrison XA. Using observation-level random effects to model overdispersion in count data in ecology and evolution. PeerJ; 2014. https://doi.org/10.7717/ peerj.616. https://www.ncbi.nlm.nih.gov/pmc/articles/PMC4194460/.

2 Peel GM D. Finite mixture models [Internet]. JohnWiley \& Sons. https: //www wiley. com/en-us/Finite+Mixture+Models-p-9780471006268; 2000. Accessed January 2, 2019, 438.

3 Grün B, Leisch F. Finite mixtures of generalized linear regression models. http ://www.springerlink.com/index/10.1007/978-3-7908-2064-5_11. Accessed January 2, 2019.

4 Dalrymple ML, Hudson IL, Ford RPK. Finite mixture, zero-inflated Poisson and hurdle models with application to SIDS. Comput Stat Data Anal. 2003;41(3-4):491-504.

5 McLachlan G. On the EM algorithm for overdispersed count data. Stat Methods Med Res. 1997;6:76-98.

6 Mufudza C, Erol H. Poisson mixture regression models for heart disease prediction. Comput Math Methods Med. 2016;2016, 4083089.

7 Joe H, Zhu R. Generalized Poisson distribution: the property of mixture of Poisson and comparison with negative binomial distribution. Biom J. 2005;47:219-229.

8 Consul PC. Generalized Poisson Distributions : Properties and Applications/. New York: M. Dekker; 1989. c.

9 Consul PC, Famoye F. Generalized Poisson regression model. Commun Stat Theor Methods. 1992;21:89-109.

10 Famoye F, Wulu JT, Singh KP. On the generalized Poisson regression model with an application to accident data. J Data Sci. 2004;2:287-295.

11 Wang W, Famoye F. Modeling household fertility decisions with generalized Poisson regression. J Popul Econ. 1997;10:273-283.

12 Maxwell RWC WL. A queuing model with state dependent service rates. J Ind Eng. 1962;12:132-136.

13 Shmueli G, Minka TP, Kadane JB, Borle S, Boatwright P. A useful distribution for fitting discrete data: revival of the Conway-Maxwell-Poisson distribution. J Roy Stat Soc: Series C (Applied Statistics). 2005;54:127-142.

14 Lord D, Guikema SD, Geedipally SR. Application of the Conway-Maxwell-Poisson generalized linear model for analyzing motor vehicle crashes. Accid Anal Prev. 2008; 40:1123-1134. 
15 Lord D, Geedipally SR, Guikema SD. Extension of the application of conwaymaxwell-Poisson models: analyzing traffic crash data exhibiting underdispersion. Risk Anal. 2010;30:1268-1276.

16 Hubert PC, Lauretto MS, Stern JM. FBST for generalized Poisson distribution. AIP Conf Proc. 2009;1193:210-217.

17 Harris T, Yang Z, Hardin JW. Modeling underdispersed count data with generalized Poisson regression. STATA J. 2012;12:736-747.

18 Lambert D. Zero-inflated Poisson regression, with an application to defects in manufacturing. Technometrics. 1992:34:1-14.

19 Rose CE, Martin SW, Wannemuehler KA, Plikaytis BD. On the use of zero-inflated and hurdle models for modeling vaccine adverse event count data. J Biopharm Stat. 2006 16:463-481.

20 Katchova A. Count Data Models Example. https://sites.google.com/site/economet ricsacademy/econometrics-models/count-data-models.

21 Atkins DC, Gallop RJ. Rethinking how family researchers model infrequent outcomes: a tutorial on count regression and zero-inflated models. J Fam Psychol. 2007;21:726-735
22 National Family Health Survey (NFHS 3). Goverment of India; 2005; 2006. http://rch iips.org/nfhs/nfhs3.shtml.

23 Yusuf OB, Ugalahi LO. On the performance of the Poisson, negative binomial and generalized Poisson regression models in the prediction of antenatal care visits in Nigeria. Am J Math Stat. 2015;5:128-136.

24 Sadia F. Performance of generalized Poisson regression model and negative binomial regression model in case of over-dispersion count data. J Emerg Technol Comput Appl Sci (IJETCAS). 2013;4:558-563.

25 Ismail N. Estimation of Claim Count Data Using Negative Binomial, Generalized Poisson, Zero-Inflated Negative Binomial and Zero-Inflated Generalized Poisson Regression Models. Spring: Casualty Actuarial Society E-Forum; 2013.

26 Hilbe JM. Negative Binomial Regression by Joseph M. Hilbe. Cambridge Core. (/core/books/negative-binomial-regression/ 12D6281A46B9A980DC6021080C9419E7). 